\title{
Les limites des inhibiteurs de points de contrôle immunitaire et la gestion de leur toxicité
}

$>$ L'immunothérapie représente désormais un des piliers de la prise en charge du cancer, notamment avec l'arrivée des inhibiteurs de points de contrôle (checkpoint) immunitaire (ICI, immune checkpoint inhibitors). Ces anticorps thérapeutiques ciblent ces co-signaux inhibiteurs entre cellules tumorales ou cellules présentatrices d'antigènes et lymphocytes $T$, activant ou réactivant ainsi une immunité cellulaire T anti-tumorale. Mais la survenue d'une toxicité immunologique, qui peut concerner tous les organes, représente le facteur limitant dans le développement clinique de ces anticorps. La gestion de cette toxicité nécessite une collaboration étroite entre oncologues et spécialistes d'organe, et repose sur l'utilisation de corticoïdes et/ou d'autres immunosuppresseurs, avec l'objectif de contrôler la dysimmunité induite sans perdre l'efficacité anti-tumorale. <

L'étude des mécanismes par lesquels les cancers échappent à l'immunovigilance a permis de révéler le rôle de diverses molécules (points de contrôle, ou checkpoints) qui inhibent la réponse immunitaire: notamment CTLA-4 (cytotoxic T-lymphocyte-associated protein 4) et PD-1 (programmed cell death 1)/ PD-LI (programmed cell death-ligand 1). Les ICI sont des anticorps thérapeutiques qui ciblent ces co-signaux inhibiteurs entre cellules tumorales ou cellules présentatrices d'antigènes et lymphocytes $T$, activant ou réactivant ainsi une immunité cellulaire $T$ anti-tumorale (Figure 1). Après leurs résultats spectaculaires dans le mélanome métastatique, puis dans les cancers du poumon inopérables, les cancers du rein et urothéliaux, certains cancers digestifs, certains lymphomes, plus d'une centaine d'essais cliniques sont actuellement en cours et l'utilisation des $\mathrm{ICl}$ est devenue l'une des stratégies thérapeutiques la plus prometteuse en oncologie. L'obtention d'une réponse tumorale stable, voire la rémission d'un cancer métastatique, se font souvent au prix d'une toxicité immunologique pouvant concerner
Marie Kostine ${ }^{1}$, Aurélien Marabelle ${ }^{2}$, Thierry Schaeverbeke', Maria Kfoury ${ }^{2}$

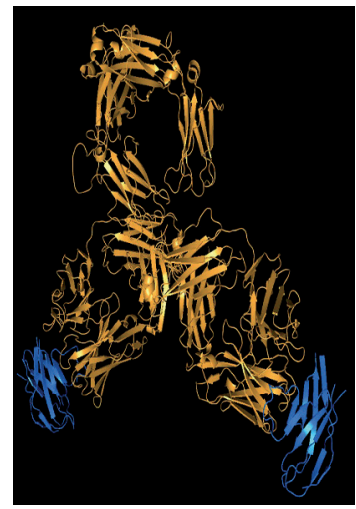

'Service de rhumatologie, hôpital Pellegrin, Bordeaux, France.

${ }^{2}$ Institut Gustave Roussy, Villejuif, France.

marie.kostine@chu-bordeaux.fr

tous les organes. Le spectre de l'auto-immunité induite par ces traitements ne cesse ainsi de s'élargir, d'autant plus qu'ils sont maintenant utilisés à grande échelle.

\section{Les effets secondaires immunologiques des inhibiteurs de points de contrôle immunitaire}

La rupture de tolérance induite par les inhibiteurs de points de contrôle immunitaire ne se limite pas à la tumeur. Ils sont susceptibles d'induire des mécanismes de dysimmunité par perte de tolérance vis-à-vis d'auto-antigènes. Et cela d'autant plus que la lyse des cellules tumorales, qu'ils induisent en ré-activant le système immunitaire, aboutit à la libération de médiateurs pro-inflammatoires et de signaux de danger (ou DAMP pour danger-associated molecular pattern). Ces complications ont été décrites sous le nom d'immunerelated adverse events (ou irAE) (Figure 2).

Ainsi, de nombreux évènements de ce type ont été observés après utilisation des anticorps anti-CTLA-4, puis avec les anticorps anti-PD-1/PD-L1 et leur combinaison [1]. Les manifestations dysimmunitaires induites diffèrent selon le type d'ICl utilisés, en raison de mécanismes d'action différents. CTLA-4 intervient lors de l'initiation de la réponse immune dans les organes lymphoïdes, au niveau des lymphocytes T naïfs, mais aussi en périphérie, au niveau des lymphocytes T régulateurs, ce qui explique le spectre large et la fréquence plus élevée d'irAE observés avec ces ICl. 


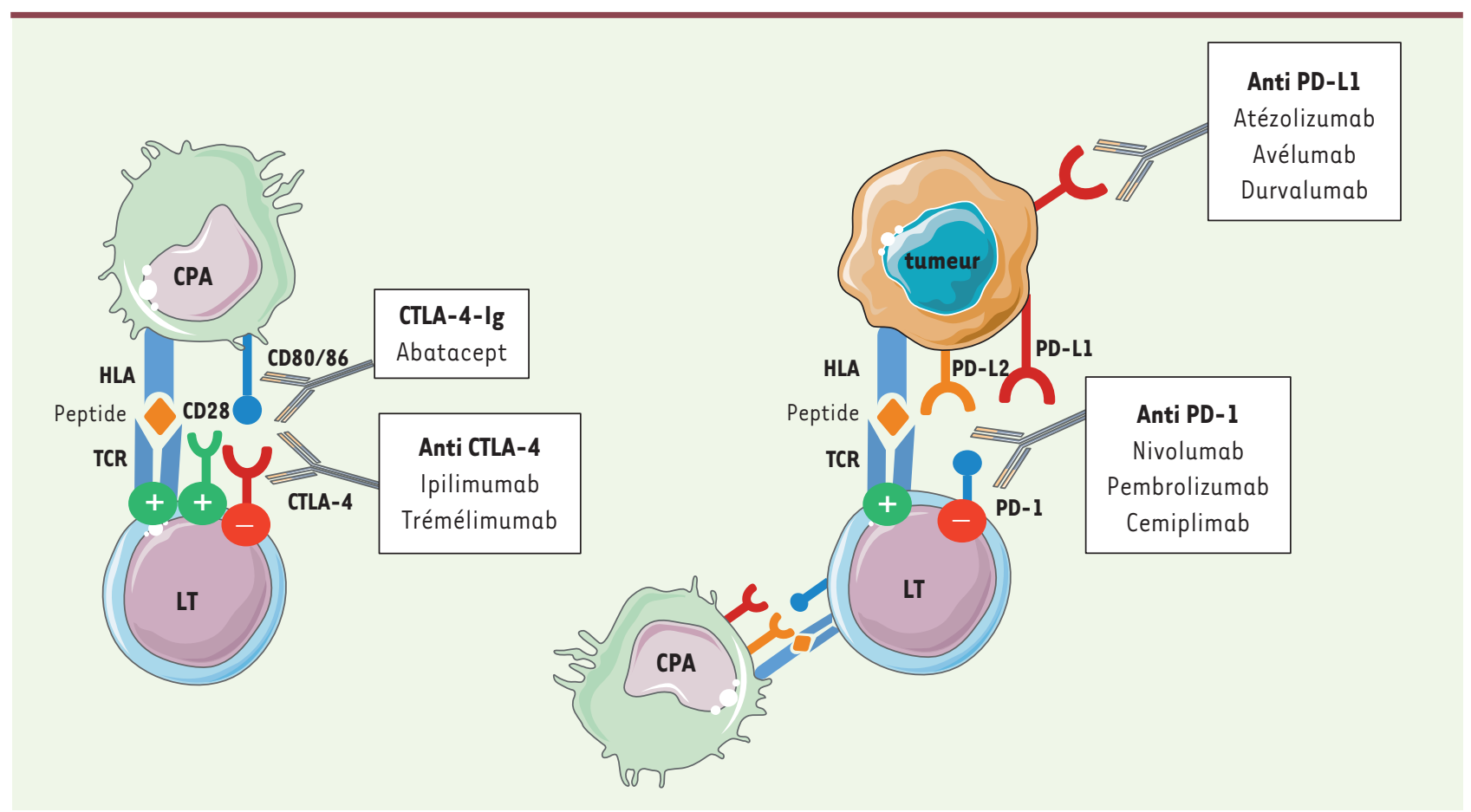

Figure 1. Principales thérapeutiques ciblant la co-stimulation.

La voie PD-1/PD-L1, quant à elle, régule l'activation lymphocytaire T au niveau périphérique ainsi qu'au sein du microenvironnement tumoral, ce qui restreint le spectre et la fréquence des irAk qui lui sont associés [2]. Cette toxicité dysimmunitaire survient généralement dans les 3 à 6 premiers mois après l'initiation du traitement. La survenue est plus précoce si des anticorps anti-CTLA-4 et anti-PD-1 sont combinés ou en cas de traitements séquentiels avec les deux anticorps [3]. Les toxicités tardives sont moins connues, en raison des périodes de suivi qui sont limitées. Bien que les irAદ puissent concerner tous les organes, les premières données de rupture de tolérance, issues des essais cliniques, ont révélé essentiellement des atteintes dermatologiques et digestives, suivies d'atteintes endocriniennes qui sont parmi les plus fréquentes [4-6]. L'usage des $\mathrm{ICl}$ en routine et la diversification de leurs indications a néanmoins permis l'identification d'irAE plus rares et de plus en plus variés.

Les complications cutanéo-muqueuses sont les plus fréquentes, observées chez plus de $40 \%$ des patients traités par immunothérapie. Elles surviennent au cours des premières semaines de traitement [7] : rash non spécifiques, souvent maculo-papuleux (tronc et extrémités), vitiligo, psoriasis, lichen et pemphigoïde (Figure 3). Des réactions sévères de type syndrome de Stevens-Johnson ${ }^{1}$, de nécrolyse épidermique, voire de syndrome d'hypersensibilité médicamenteuse (en anglais DRESS, pour drug reaction [ou rash] with eosinophilia and systemic symptoms) ont également été rapportées.

Les complications digestives sont également très fréquentes, avec des diarrhées observées chez $30 \%$ des patients. D’authentiques colites

${ }^{1}$ Le syndrome de Stevens-Johnson est une forme de nécrolyse épidermique caractérisée par la destruction et le détachement de l'épithélium de la peau et des muqueuses inflammatoires peuvent survenir, identiques à celles observées dans la maladie de Crohn ou les colites microscopiques [8]. Elles mettent en jeu le pronostic vital du patient en raison du risque de perforation intestinale. Les colites s'observent principalement avec les anticorps anti-CTLA-4, en monothérapie ou en traitement combiné, avec une médiane d'apparition de 8 semaines après l'introduction de I'ICI [9]. Les analyses histologiques et immunologiques des biopsies ont montré une différence entre les colites induites sous traitement par anticorps anti-CTLA-4 (infiltrat T CD4 ${ }^{+}$, forte sécrétion de TNF- $\alpha$ ) et celles sous anticorps antiPD-1 (infiltrat T CD8 $8^{+}$, forte proportion de lymphocytes $T$ régulateurs au sein de la population ICOS[inducible $T$-cell costimulator $\left.]^{+} \mathrm{CD} 4^{+}\right)$[10]. Un cas de maladie cœliaque a été rapporté sous ipilimumab, dans un contexte de diarrhées résistantes aux corticoïdes [11]. Les complications hépatiques sous $\mathrm{ICl}$ concernent environ $3,5 \%$ des patients. Les biopsies hépatiques ont montré des différences histologiques selon la molécule utilisée, avec une atteinte granulomateuse sous anticorps anti-CTLA-4 et lobulaire sous anticorps anti-PDL1 [12]. Des cas d'augmentation du taux de lipase et d'authentiques pancréatites ont également été rapportés, là aussi plus fréquemment sous anti-CTLA-4 ou lors de combinaisons d'ICI [13].

Les complications endocriniennes concernent principalement la thyroïde et I'hypophyse (Figure 4), mais la 


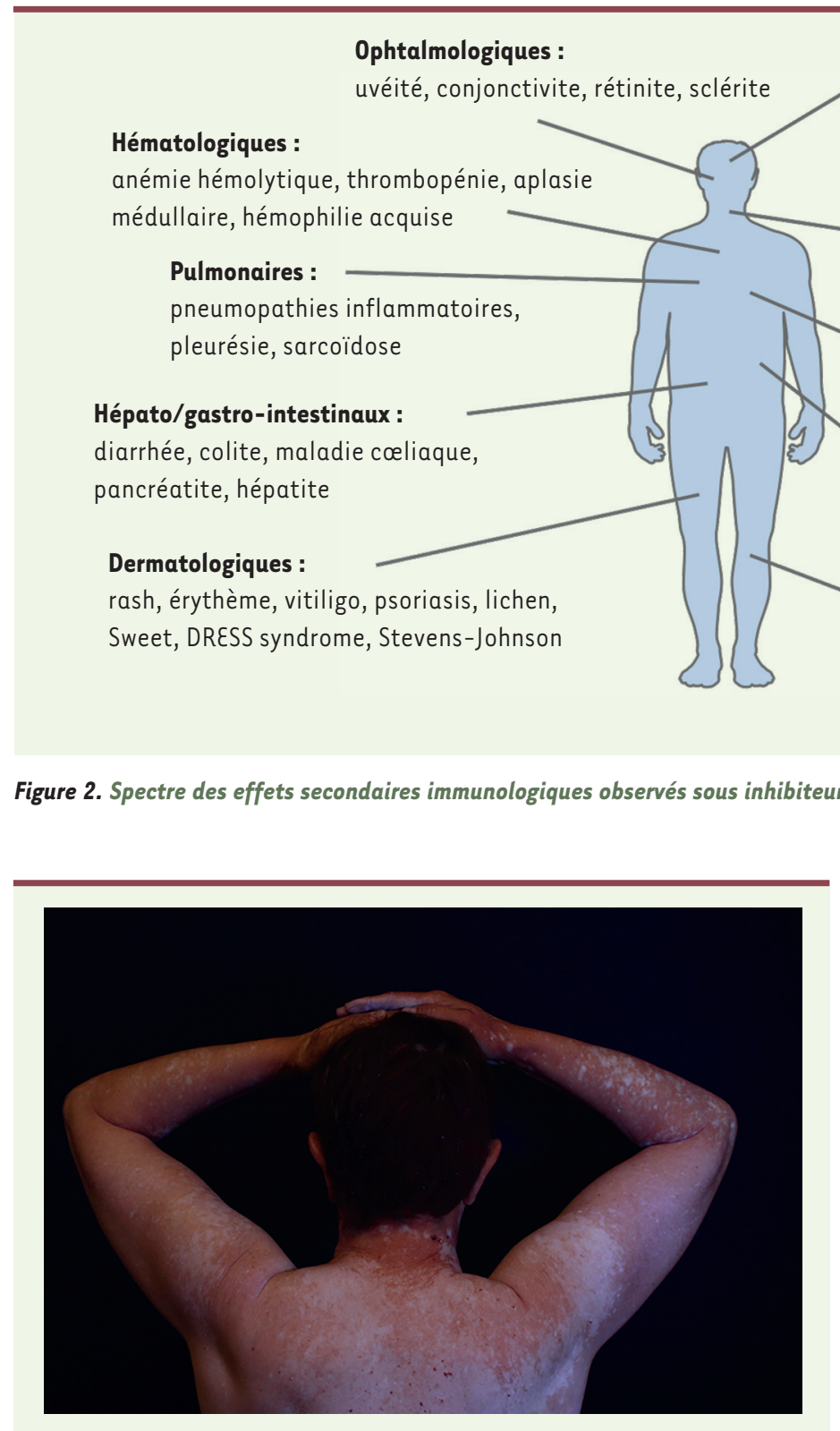

Figure 3. Vitiligo induit sous traitement anti-PD-1 reçu pour un mélanome métastatique (collection J. Seneschal, Bordeaux, France).

traduction clinique de ces atteintes aspécifiques (asthénie, faiblesse musculaire) rend le diagnostic difficile. Les hypophysites autoimmunes font partie des complications endocriniennes rapportées, principalement sous anticorps anti-CTLA-4, rares sous anticorps anti-PD-1. Elles présentent les mêmes caractéristiques cliniques, biologiques et radiologiques qu'une hypophysite lymphocytaire, une pathologie auto-immune rare observée pendant la grossesse ou en post-partum. La physiopathologie des hypophysites auto-immunes repose sur la reconnaissance directe des cellules hypophysaires, qui expriment CTLA-4, par les anticorps anti-CTLA-4, accompagné par une activation du complément [14]. L'atteinte thyroïdienne est plus

\section{Neurologiques :}

encéphalite, myélite, méningite aseptique, Guillain-Barré, myasthénie, neuropathie périphérique

\section{Endocrinologiques :}

hypophysite, dysthyroïde, diabète, insuffisance surrénalienne

\section{Cardio-vasculaires :}

myocardite, péricardite, vascularite

\section{Néphrologiques:}

néphropathie lupique, glomérulonéphrite interstitielle granulomateuse

\section{Rhumatologiques :}

arthralgie, myalgie, polyarthrite, pseudo-polyarthrite rhizomélique, lupus, sclérodermie, syndrome sec, myosite, vascularite

de points de contrôle immunitaire.

fréquemment observée sous anticorps anti-PD-1 ou en combinaison, avec une prédominance d'hypothyroïdie. Des hyperthyroïdies ont été également rapportées, rarement d'authentiques maladies de Basedow². Plus rarement, une insuffisance surrénalienne primitive ou un diabète insulino-dépendant se développent sous une forme fulminante. II est intéressant de souligner que la majorité des cas de diabète surviennent chez des patients HLA(human leukocyte antigen)-DR4 dont les sérums, avant traitement, contiennent dans plus de la moitié des cas des auto-anticorps, soulignant une auto-immunité pré-existante [15].

Les complications pulmonaires sont rares, environ $5 \%$ des patients sous traitement par anticorps anti-PD-Ll et $10 \%$ en cas de combinaison, mais potentiellement graves. Les aspects radiologiques sont variables et non spécifiques : pneumopathie organisée cryptogénique (POC), verre dépoli isolé, pneumopathie interstitielle non spécifique (NSIP) et pneumopathie d'hypersensibilité (PHS) [16]. La dyspnée et la toux sont les points d'appel cliniques. Cependant un tiers des patients reste asymptomatique avec une découverte fortuite du fait d'une imagerie réalisée dans le cadre du suivi oncologique. De nombreux cas de sarcoïdose sont également rapportés sous ICI. Les patients sont asymptomatiques dans $25 \%$ des cas et la découverte fortuite à l'imagerie de suivi oncologique d'adénopathies médiastinales et/ou de nodules pulmonaires souvent hypermétaboliques, suggèrent une

\footnotetext{
2 hyperthyroïdie auto-immune.
} 


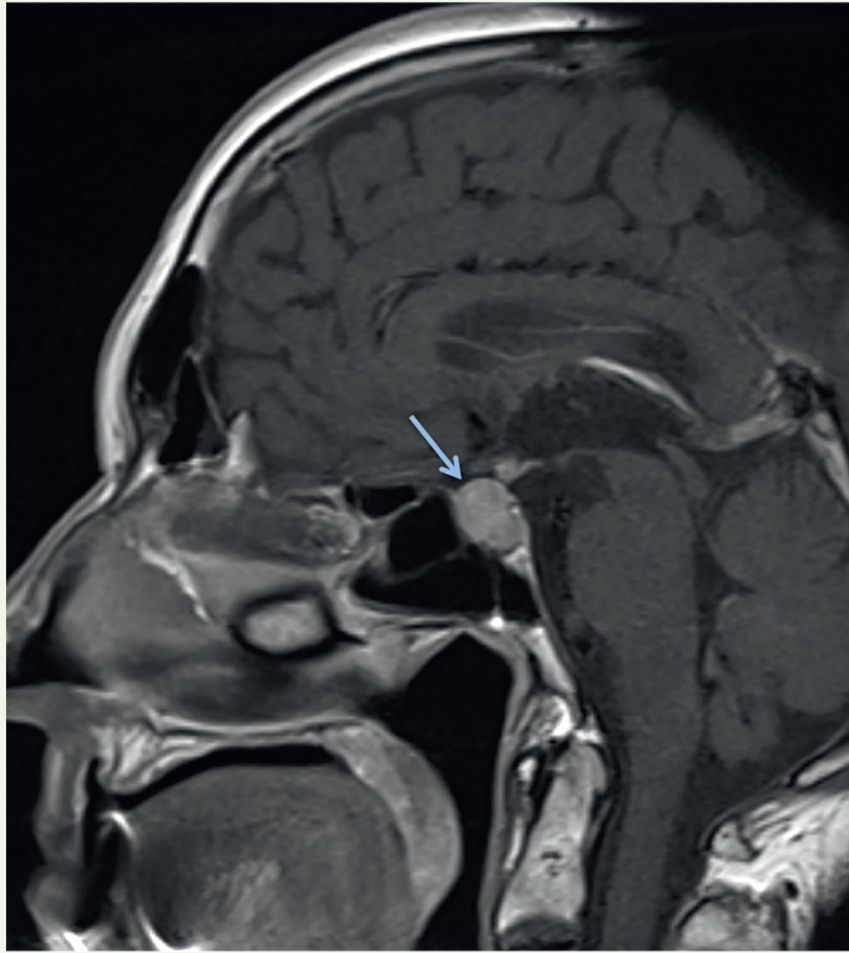

Figure 4. Hypophysite auto-immune apparue sous anticorps anti-CTLA-4 (collection M. Haissaguerre, Bordeaux, France).

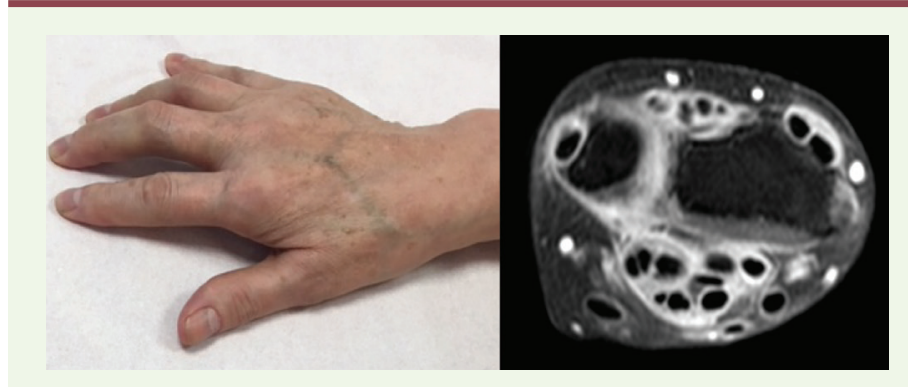

Figure 5. Synovite radio-ulnaire distale et ténosynovites des fléchisseurs et extenseurs observée sous traitement anti-PD-1.

progression tumorale. La documentation histologique est alors d'un intérêt majeur avant d'envisager l'arrêt du traitement par ICI [17].

Les complications rhumatologiques sont peu fréquentes (6-7\%) mais très variées : arthralgies et myalgies, mono-oligoarthrite, polyarthrite, pseudo-polyarthrite rhizomélique, rhumatisme psoriasique, syndrome RS3PE (remitting seronegative symmetrical synovitis with pitting edema, ou polyarthrite aiguë œdémateuse bénigne du sujet âgé), syndrome sec, vascularite, lupus, sclérodermie et myosite (Figure 5). Dans certains cas il s'agit d'une authentique pathologie rhumatologique ou systémique (Gougerot-Sjögren ${ }^{3}$, PR [polyarthrite rhuma-

${ }^{3}$ Le syndrome de Gougerot Sjögren est une maladie auto-immune caractérisée par une infiltration lymphoïde des glandes salivaires et lacrymales responsable d'une sécheresse buccale et oculaire. toïde], vascularite à ANCA [auto-anticorps anti-cytoplasmiques de neutrophiles], etc.) mais la majorité des patients ne remplissent pas les critères de classification et présentent donc plutôt des pathologies rhumatologiques-like. La négativité du syndrome inflammatoire biologique ou du bilan immunologique ne doit donc pas exclure le diagnostic d'un irAE rhumatologique/ systémique. Les myosites sont fréquemment associées à une myasthénie ( $15 \%$ ) ou une myocardite. La myasthénie doit être évoquée devant un ptosis (chute de la paupière supérieure), une diplopie ${ }^{4}$ et/ou une symptomatologie bulbaire.

Les complications neurologiques sont rares ( $1 \%)$ et très variées, allant de la céphalée modérée à l'encéphalite grave [18]. Parmi les toxicités neurologiques, ont été décrits : syndrome de Guillain-Barré, myélite, méningite aseptique, myopathie inflammatoire, myasthénie (possiblement associée à une myosite) et neuropathie périphérique.

Les complications cardiologiques sont potentiellement fatales, isolées ou compliquant certaines myosites. II s'agit principalement de la myocardite, à rechercher absolument en cas de suspicion, par un dosage de la troponine, un électro-cardiogramme, voire une échographie ou une IRM (imagerie par résonnance nucléaire) cardiaque [19]. Elle survient habituellement dans les six premières semaines de traitement par $\mathrm{ICl}$ et peut se révéler par une dyspnée ou une douleur thoracique.

Les complications rénales concernent 1 à $2 \%$ des patients sous $\mathrm{ICl}$ en monothérapie et jusqu'à $5 \%$ des patients recevant une combinaison [20]. Il s'agit principalement de néphrites tubulo-interstitielles aiguës, mais une atteinte granulomateuse est parfois retrouvée. Une néphrite lupique a été rapportée sous ipilimumab.

Les complications plus rares. II s'agit de complications ophtalmologiques et hématologiques (moins de $1 \%$ ). Quelques études ont ainsi rapportés des manifestations ophtalmologiques inflammatoires induites sous $\mathrm{ICl}$ : uvéite, sclérite, rétinite, orbitopathie inflammatoire, voire une maladie de Vogt-Koyanagi-Harada ${ }^{5}$. Une étude française prospective a évalué à $0,4 \%$ la prévalence des irAE ophtalmologiques [21]. Des irAE hématologiques ont également été décrits : principalement des neutropénies, anémies hémolytiques ou thrombopénies auto-immunes et anémies aplasiques [22]. Une hémophilie A a aussi été rapportée sous ipilimumab.

\footnotetext{
Perception visuelle double d'un objet unique.

${ }^{5}$ Uvéo-méningite bilatérale d'origine auto-immune.
} 


\section{Effets secondaires immunologiques des inhibiteurs de points de contrôle immunitaire dans des populations particulières}

Maladie inflammatoire préexistante. Du fait d'un risque théorique d'aggravation de la maladie inflammatoire ou auto-immune, les patients présentant une maladie inflammatoire préexistante ont été exclus des essais cliniques. Nous disposons néanmoins de plusieurs séries rétrospectives révélant qu'environ la moitié des patients ont présenté soit des irAદ classiques, soit des exacerbations de la pathologie inflammatoire préexistante, généralement bien contrôlés par une corticothérapie ou par l'optimisation du traitement immuno-modulateur [23, 24]. Les données actuelles ne suggèrent pas de contreindication aux $\mathrm{ICl}$ pour les patients avec une maladie inflammatoire préexistante, mais des études prospectives de plus larges effectifs sont nécessaires pour valider ces observations rétrospectives.

Malades transplantés. Ces patients nécessitent une modulation étroite de l'immunosuppression pour maintenir une tolérance du greffon qu'ils ont reçu et éviter un rejet ou une réaction du greffon contre l'hôte. L'anticorps anti-CTLA-4, bien qu'ayant une fréquence et un spectre de toxicité plus large, semble mieux toléré dans cette population que l'anticorps anti-PD-1 [25,26]. Des données précliniques viennent conforter cette observation en montrant le rôle majeur de la voie PD-1/ PD-Ll dans la tolérance du greffon [27]. Une revue de la littérature récente rapporte un rejet du greffon solide chez $41 \%$ des patients : $11 / 23$ patients avec greffe rénale, 4/11 patients avec greffe hépatique et $1 / 5$ patients avec greffe cardiaque, sans différence entre les patients traités par l'anticorps anti-CTLA-4 et ceux traités par l'anticorps antiPD-1, et avec délai médian de 21 jours [28]. La prise en charge oncologique optimale reste donc à déterminer dans cette population.

Maladie infectieuse chronique (exemple du VIH). Sauf dans de rares exceptions, les infections virales par le VIH (virus de l'immunodéficience humaine), le VHB et le VHC (virus de l'hépatite B et C) sont également des critères d'exclusion des essais cliniques sur les $\mathrm{ICl}$. L'idée sous-jacente est que ces infections chroniques pourraient altérer la fonction lymphocytaire $T$ et donc, théoriquement, compromettre l'efficacité de I'ICI, surtout chez les patients infectés par le VIH qui présentent un taux de lymphocytes T $C D 4^{+}$diminué. En réalité, les données rétrospectives disponibles sont extrêmement rassurantes, sans signal d'alerte sur la toxicité (avec 8,6\% d'irA\& sévères), une charge virale restant indétectable ( $93 \%$ des cas) et même une augmentation du nombre de lymphocytes $\mathrm{CD}^{+}$[29]. Des essais cliniques évaluant un traitement par anticorps anti-PD-1 en monothérapie (NCT02595866, NCT03304093) ou en combinaison avec l'anticorps anti-CTLA-4 (NCT02408861) sont en cours. En attente des résultats de ces essais, il est considéré qu'une infection virale chronique, notamment par le VIH, ne représente pas de contre-indication à l'immunothérapie.

\section{La gestion des toxicités}

La prise en charge des irAE repose sur leur identification précoce et une collaboration étroite entre oncologue référent et spécialiste de l'organe touché [30]. La définition des grades de sévérité, par symptôme et par organe, est fondée sur la classification common terminology criteria for adverse events (CTCAE) du National Cancer Institute, disponible en ligne ${ }^{6}$. Pour certaines toxicités, cette classification mérite d'être réévaluée régulièrement [31]. La prise en charge des irAE s'appuie sur le grade défini par cette classification et les stratégies thérapeutiques reposent sur les protocoles de gestion des toxicités qui ont été développés par les laboratoires pharmaceutiques au cours des essais cliniques.

\section{Les recommandations actuelles}

II existe actuellement trois recommandations internationales pour la gestion des irAE :

- Les recommandations de l'ESMO (European society of medical oncology) [3]

- Les recommandations de la SITC (Society for immunotherapy of cancer) [32]

- Les recommandations de l'ASCO (American society of clinical oncology) [33]

Ces recommandations ont été développées principalement par des oncologues. S'y ajoutent des recommandations spécifiques des sociétés savantes, telles que les recommandations pour la gestion des irAk endocriniens [34] ou des irAE rhumatologiques.

Pour les irAE de grade 1, le traitement symptomatique est privilégié et la corticothérapie n'est pas indiquée.

La corticothérapie est le traitement de $1^{\text {re }}$ intention pour les irAE de grade 2. La dose recommandée varie entre 0,5 et $1 \mathrm{mg} / \mathrm{Kg}$ selon chaque type d'irAc. Par exemple, une dose inférieure ou égale à $20 \mathrm{mg} / \mathrm{j}$ d'équivalent du corticoïde prednisone est souvent suffisante pour les irAE rhumatologiques, en complément d'éventuels gestes locaux, si besoin est.

Pour les irAE de grade 3, notamment dermatologiques, digestifs, pulmonaires, rénaux, hématologiques et neurologiques, la corticothérapie proposée est de 1 à $2 \mathrm{mg}$ / $\mathrm{Kg}$ par voie intraveineuse. Des discussions persistent dans les cas d'hépatites immuno-induites: des résolutions spontanées étant observées, la corticothérapie systématique est donc souvent débattue [35].

Les autres traitements immunosuppresseurs. Le recours à d'autres traitements immunosuppresseurs (traitements conventionnels ou biologiques) peut être nécessaire en cas de persistance ou d'aggravation des irAk sous corticothérapie, ou en cas de corticorésistance ou de corticodépendance. Les molécules utilisées dépendent du type d'irAc : le méthotrexate,

${ }^{6}$ https://ctep.cancer.gov/protocoldevelopment/electronic_applications/docs/ CTCAE_v5_Puick_Reference_5x7.pdf. 
I'hydroxychloroquine et la salazopyrine sont les traitements conventionnels les plus utilisés pour les irAz rhumatologiques, avec un possible sur-risque de réaction d'hypersensibilité pour la salazopyrine dans cette situation [36-38]. Le mycophénolate mofétil (un immunosuppresseur) est également utilisé dans certains cas d'hépatites, pneumopathies immuno-induites, myosites et sclérodermie-like [39]. Fondé sur l'idée d'un parallèle avec les réactions de greffon contre I'hôte, le tacrolimus a récemment été testé dans des situations d'irAદ ( 2 colites et 1 éruption sévère avec atteinte muqueuse) en échec de la corticothérapie et d'un traitement par anticorps anti-TNF- $\alpha$ [40]. L'infliximab a été le premier traitement biologique utilisé dans le traitement des colites sévères par les gastro-entérologues. Les données concernant les anticorps anti-TNF- $\alpha$ sont rassurantes pour un usage ponctuel (1 ou 2 perfusions), mais peu de données existent sur leur utilisation au long cours. Les équipes de rhumatologie américaines ont également utilisé fréquemment les anticorps anti-TNF- $\alpha$ en cas d'échec de la corticothérapie. Un essai clinique oncologique de phase I associant ICl et anti-TNF- $\alpha$ est en cours (NCT03293784). Il devrait apporter des informations sur l'efficacité de ce traitement et les risques de cette association, utiles pour notre gestion des irAદ sévères. Certains patients ont reçu du tocilizumab pour des irAE variés, soit à la dose de $4 \mathrm{mg} / \mathrm{kg}$ par voie intra-veineuse, avec une amélioration dans $80 \%$ des cas, soit à la dose de $162 \mathrm{mg}$ bimensuel par voie sous-cutanée pour des atteintes rhumatologiques [41]. Le rituximab a lui aussi été utilisé, avec des schémas thérapeutiques différents, chez des patients présentant des irAE hématologiques ou neurologiques [42]. Une étude de phase II, associant rituximab et anticorps anti-PD-1 chez des patients présentant un lymphome folliculaire a montré une bonne efficacité et une bonne tolérance [43]. Enfin, le védolizumab, un AcM anti-intégrine $\alpha 4-\beta 7$, approuvé dans les maladies inflammatoires chroniques intestinales, qui agit en limitant le recrutement lymphocytaire dans le tractus gastro-intestinal, a aussi été utilisé chez des patients présentant une colite immunoinduite réfractaire [44]. Le seul signal négatif provient à ce jour de l'anticorps anti-IL(interleukine)-17, le sécukinumab, utilisé pour le traitement d'une poussée d'une maladie de Crohn, un psoriasis sévère et des arthralgies observées sous traitement par l'anticorps anti-PD-1. II a permis une résolution complète des irAE au prix de la perte de l'efficacité anti-tumorale initiale [45]. Une publication récente rapporte l'utilisation de l'anticorps anti-IL-17 dans la gestion d'une atteinte psoriasique immuno-induite sans altération de la réponse anti-tumorale.

II n'existe pas de données montrant que les patients ayant reçu de tels traitements pour la gestion d'un irAk aient une moins bonne réponse tumorale. Toutefois, l'impact à long terme de la corticothérapie et des autres médicaments immunosuppresseurs dans le cadre du cancer devra être étroitement surveillé.

\section{Poursuite du traitement oncologique}

Le traitement oncologique par $\mathrm{ICl}$ peut être poursuivi pour les irAE de grade 1, sous réserve d'une surveillance rapprochée, et avec une vigilance particulière pour les irAE neurologiques, hématologiques et cardiologiques. Pour les irAદ de grade 2 , il est souvent conseillé de sus- pendre temporairement le traitement jusqu'à résolution ou retour à un grade 1 . Pour les irAc de grade 3, l'arrêt de I'ICl est indiqué, au moins de façon temporaire, parfois définitif. Sa reprise se discutera lorsque l'irAE sera résolu ou après un retour à un grade 1 . En cas d'irAE de grade 4, I'ICl sera définitivement arrêté, à l'exception des endocrinopathies. Les endocrinopathies sont en effet le plus souvent irréversibles et devront être supplémentées à vie. La survie des patients qui arrêtent le traitement par $\mathrm{ICl}$ en raison d'un irAE n'est toutefois pas altérée [46].

\section{Stratégies de prévention}

Devant la fréquence des diarrhées et colites que I'on observe sous traitement par anticorps anti-CTLA-4, une prophylaxie par budésonide a été testée, sans démonstration d'efficacité [47]. Les patients présentant une maladie auto-immune, théoriquement plus à risque de développer des irAE, sont également concernés, afin de contrôler la maladie auto-immune dont ils sont atteints avant le début du traitement par I'ICl et afin de diminuer le risque de poussée et/ou d'irAk. Une étude rétrospective multicentrique française ayant inclus 112 patients avec maladie auto-immune ou inflammatoire préexistante (principalement psoriasis, PR, maladie inflammatoire chronique de l'intestin, spondylarthrite ankylosante, lupus) a montré une survie sans progression plus courte et un taux de réponse inférieur chez les patients recevant un traitement immunosuppresseur à l'instauration de I'ICI [48]. De même, il a récemment été rapporté qu'une corticothérapie supérieure à $10 \mathrm{mg} / \mathrm{j}$ d'équivalent prednisone au début du traitement par l'ICl était associée à une réponse anti-tumorale et des taux de survie plus faibles [49].

$\varepsilon n$ attendant des études prospectives confirmant ou non ces données, il est prudent d'essayer de minimiser le traitement par corticoïdes et immunosuppresseurs au moment de l'introduction de l'ICl. Plusieurs groupes ont démontré que la survenue d'irAદ était associée à une meilleure réponse à $|'| C \mid$, suggérant que cette auto-immunité pourrait interférer avec l'immunité anti-tumorale [50]. Il ne semble donc pas nécessaire de prévenir les irAE chez tous les patients, mais il est essentiel d'identifier ceux à risque d'irAk sévères, afin d'anticiper la survenue de ces évènements et d'adapter les stratégies de prévention et de prise en charge.

\section{Conclusion}

La connaissance des irAk est essentielle pour l'ensemble de la communauté médicale qui sera confrontée à la 
prise en charge des patients traités par ICl. La prise en charge des irAE reste un défi car il est essentiel de contrôler la toxicité immunologique sans perdre l'efficacité anti-tumorale, et la stratégie optimale reste à déterminer. La prise en charge des toxicités, plus fréquentes lors des combinaisons de traitements, est certainement un des enjeux majeurs du développement de l'immunothérapie. $\diamond$

\section{SUMMARY}

Toxicities of immune checkpoint inhibitors and their management Immunotherapeutic strategies, notably immune checkpoint inhibitors, have become a standard of care for the treatment of advanced cancers, with a growing spectrum of activity.

These monoclonal antibodies target the co-inhibitory signals between tumor cells or antigen-presenting cells and T cells, thereby enhancing antitumour $T$ cell activity. However, the occurrence of immune-related adverse events, that can affect all organ-system, represents a major limiting factor to the clinical development of these antibodies. Management of such toxicity requires a close collaboration between oncologists and organ-specialists, by using glucocorticoids and/or other immunosuppressive therapies, with the common objective not alter anti-tumor response. $\diamond$

\section{LIENS D'INTÉRÊT}

Les auteurs déclarent n'avoir aucun lien d'intérêt concernant les données publiées dans cet article.

\section{RÉFÉRENCES}

1. Boutros C, Tarhini A, Routier $\varepsilon$, et al. Safety profiles of anti-CTLA-4 and anti-PD-1 antibodies alone and in combination. Nat Rev Clin Oncol 2016 ; $13: 473-86$.

2. Pardoll DM. The blockade of immune checkpoints in cancer immunotherapy. Nat Rev Cancer 2012 ; $12: 252-64$.

3. Haanen JBAG, Carbonnel F, Robert C, et al. Management of toxicities from immunotherapy: ESMO Clinical Practice Guidelines for diagnosis, treatment and follow-up. Ann Oncol 201829 (suppl 4) : iv264-i6.

4. Belum VR, Benhuri B, Postow MA, et al. Characterisation and management of dermatologic adverse events to agents targeting the PD-1 receptor. Eur J Cancer 2016; 60 : 12-25.

5. Cramer P, Bresalier RS. Gastrointestinal and Hepatic Complications of Immune Checkpoint Inhibitors. Curr Gastroenterol Rep $2017 ; 19: 3$.

6. Byun DJ, Wolchok JD, Rosenberg LM, Girotra M. Cancer immunotherapy - immune checkpoint blockade and associated endocrinopathies. Nat Rev Endocrinol 2017 ; 13 : 195-207.

7. Sibaud V, Boulinguez $S$, Pagès $C$, et al. Dermatologic toxicities of immune checkpoint inhibitors. Ann Dermatol Venereol 2018 ; 145 : 313-30.

8. Bertha M, Bellaguara $\varepsilon$, Kuzel T, Hanauer $S$. Checkpoint inhibitor-induced colitis: A new type of inflammatory bowel disease? ACG Case Rep J 2017 ; 4 : ell2.

9. Collins M, Michot JM, Danlos FX, et al. Inflammatory gastrointestinal diseases associated with PD-1 blockade antibodies. Ann Oncol $2017 ; 28: 2860-5$.

10. Coutzac C, Adam J, Soularue $\varepsilon$, et al. Colon immune-related adverse events: Anti-CTLA-4 and anti-PD-1 blockade induce distinct immunopathological entities. J Crohns Colitis 2017 ; 11 : 1238-46.

11. Gentile NM, D'Souza A, Fujii LL, et al. Association between ipilimumab and celiac disease. Mayo Clin Proc $2013 ; 88: 414-7$.

12. De Martin $\varepsilon$, Michot J-M, Papouin B, et al. Characterization of liver injury induced by cancer immunotherapy using immune checkpoint inhibitors. J Hepatol 2018; 68 : 1181-90.

13. Su $Q$, Zhang $X-C$, Zhang $C-G$, et al. Risk of immune-related pancreatitis in patients with solid tumors treated with immune checkpoint inhibitors: Systematic assessment with meta-analysis. J Immunol Res $2018 ; 2018: 1027323$.

14. Postow MA, Sidlow R, Hellmann MD. Immune-related adverse events associated with immune checkpoint blockade. N Engl J Med 2018 ; 378 : 158-68.

15. Stamatouli AM, Quandt Z, Perdigoto AL, et al. Collateral damage: insulin-dependent diabetes induced with checkpoint inhibitors. Diabetes $2018 ; 67: 1471-80$.

16. Naidoo J, Wang $X$, Woo KM, et al. Pneumonitis in patients treated with anti-programmed death-1/ programmed death ligand 1 therapy. J Clin Oncol 2017 ; 35 : 709-17.
17. Firwana B, Ravilla R, Raval M, et al. Sarcoidosis-like syndrome and lymphadenopathy due to checkpoint inhibitors. J Oncol Pharm Pract 2017 ; $23: 620-4$

18. Larkin J, Chmielowski B, Lao CD, et al. Neurologic serious adverse events associated with nivolumab plus ipilimumab or nivolumab alone in advanced melanoma, including a case series of encephalitis. Oncologist $2017 ; 2$ : 709-18.

19. Moslehi JJ, Salem J- $\varepsilon$, Sosman JA, et al. Increased reporting of fatal immune checkpoint inhibitor-associated myocarditis. Lancet 2018;391:933.

20. Murakami N, Motwani S, Riella LV. Renal complications of immune checkpoint blockade. Curr Probl Cancer 2017 ; $41: 100-10$.

21. Bitton K, Michot J-M, Barreau E, et al. Prevalence and clinical patterns of ocular complications associated with anti-PD-1/PD-LI anticancer immunotherapy. Am J Ophthalmol $2019 ; 202$ : 109-17.

22. Delanoy N, Michot J-M, Comont T, et al. Haematological immune-related adverse events induced by anti-PD-1 or anti-PD-L1 immunotherapy: a descriptive observational study. Lancet Haematol $2019 ; 6$ : e48-57.

23. Menzies AM, Johnson DB, Ramanujam S, et al. Anti-PD-1 therapy in patients with advanced melanoma and preexisting autoimmune disorders or major toxicity with ipilimumab. Ann Oncol $2017 ; 28$ : 368-76.

24. Johnson DB, Khushalani NI, Puzanov I, et al. Ipilimumab in metastatic melanoma patients with pre-existing autoimmune disorders. J Clin Oncol 2017 ; 33 (suppl) : 9019.

25. Davids MS, Kim HT, Bachireddy P, et al. Ipilimumab for patients with relapse after allogeneic transplantation. N Engl J Med 2016 ; 375 : 143-53.

26. Lipson $\varepsilon$ J, Bagnasco SM, Moore J, et al. Tumor regression and allograft rejection after administration of anti-PD-1. N Engl J Med 2016 ; 374 : 896-8.

27. Tanaka K, Albin MJ, Yuan X, et al. PDLl is required for peripheral transplantation tolerance and protection from chronic allograft rejection. J Immunol 2007 ; 179 : 5204-10.

28. Abdel-Wahab N, Safa H, Abudayyeh A, et al. Checkpoint inhibitor therapy for cancer in solid organ transplantation recipients: an institutional experience and a systematic review of the literature. J Immunother Cancer $2019 ; 7: 106$.

29. Cook MR, Kim C. Safety and efficacy of immune checkpoint inhibitor therapy in patients with HIV infection and advanced-stage cancer: a systematic review. JAMA Oncol 2019 ; 5: 1049-54.

30. Champiat S, Lambotte 0 , Barreau $\varepsilon$, et al. Management of immune checkpoint blockade dysimmune toxicities: a collaborative position paper. Ann Oncol $2016 ; 27: 559-74$.

31. Friedman CF, Clark V, Raikhel AV, et al. Thinking critically about classifying adverse events: incidence of pancreatitis in patients treated with nivolumab + ipilimumab. J Natl Cancer Inst 2017 ; 109. doi: 10.1093/jnci/djw260.

32. Puzanov I, Diab A, Abdallah K, et al. Managing toxicities associated with immune checkpoint inhibitors: consensus recommendations from the Society for immunotherapy of cancer (SITC) toxicity management working group. J Immunother Cancer $2017 ; 5$ : 95.

33. Brahmer JR, Lacchetti C, Schneider BJ, et al. Management of immunerelated adverse events in patients treated with immune checkpoint inhibitor therapy: American society of clinical oncology clinical practice guideline. J Clin Oncol $2018 ; 36: 1714-68$.

34. Castinetti F, Albarel F, Archambeaud F, et al. French Endocrine society guidance on endocrine side-effects of immunotherapy. Endocr Relat Cancer 2018 0ct l; pii : ERC-18-0320.Rl.

35. Gauci M-L, Baroudjian B, Zeboulon C, et al. Immune-related hepatitis with immunotherapy: are corticosteroids always needed? J Hepatol 2018; 69 : 548-50.

36. Kostine M, Rouxel L, Barnetche T, et al. Rheumatic disorders associated with immune checkpoint inhibitors in patients with cancer-clinical aspects and relationship with tumour response: a single-centre prospective cohort study. Ann Rheum Dis 2017 ; 77 : 393-8.

37. Roberts J, Smylie M, Walker J, et al. Hydroxychloroquine is a safe and effective steroid-sparing agent for immune checkpoint inhibitor-induced inflammatory arthritis. Clin Rheumatol 2019 ; $38: 1513-9$.

38. Ford M, Sahbudin I, Filer A, et al. High proportion of drug hypersensitivity reactions to sulfasalazine following its use in anti-PD-1-associated inflammatory arthritis. Rheumatology 2018 ; 57 : 2244-6.

39. Friedman CF, Proverbs-Singh TA, Postow MA. Treatment of the immunerelated adverse effects of immune checkpoint inhibitors: a review. JAMA Oncol 2016; 2 : 1346-53.

40. Beardslee T, Draper A, Kudchadkar R. Tacrolimus for the treatment of immune-related adverse effects refractory to systemic steroids and antitumor necrosis factor $\alpha$ therapy. J Oncol Pharm Pract $2019 ; 25: 1275-81$. 


\section{RÉFÉRENCES}

41. Stroud CR, Hegde A, Cherry C, et al. Tocilizumab for the management of immune mediated adverse events secondary to PD-1 blockade. J Oncol Pharm Pract $2019 ; 25$ : 551-7.

42. Khan U, Ali F, Khurram MS, et al. Immunotherapy-associated autoimmune hemolytic anemia. J Immunother Cancer $2017 ; 5: 15$.

43. Westin JR, Chu F, Zhang M, et al. Safety and activity of PDI blockade by pidilizumab in combination with rituximab in patients with relapsed follicular lymphoma: a single group, open-label, phase 2 trial. Lancet Oncol $2014 ; 15: 69--77$.

44. Bergqvist V, Hertervig $\varepsilon$, Gedeon P, et al. Vedolizumab treatment for immune checkpoint inhibitorinduced enterocolitis. Cancer Immunol Immunother 2017 ; 66 : 581-92.

45. Esfahani K, Miller WH. Reversal of autoimmune toxicity and loss of tumor response by interleukin-17 blockade. N Engl J Med 2017 ; 376 : 1989-91.

46. Schadendorf D, Wolchok JD, Hodi FS, et al. Efficacy and safety outcomes in patients with advanced melanoma who discontinued treatment with nivolumab and ipilimumab because of adverse events: a pooled analysis of randomized phase II and III trials. J Clin Oncol $2017 ; 35: 3807-14$
47. Weber J, Thompson JA, Hamid 0, et al. A randomized, double-blind, placebocontrolled, phase II study comparing the tolerability and efficacy of ipilimumab administered with or without prophylactic budesonide in patients with unresectable stage III or IV melanoma. Clin Cancer Res 2009; $15: 5591-8$.

48. Tison A, Quéré G, Misery L, et al. Safety and efficacy of immune checkpoint inhibitors in patients with cancer and preexisting autoimmune diseases: a nationwide multicenter retrospective study. Arthritis Rheum 2019. doi: 10.1002/art.41068

49. Arbour KC, Mezquita L, Long N, et al. Impact of baseline steroids on efficacy of programmed cell death- 1 and programmed death-ligand 1 blockade in patients with non-small-cell lung cancer. J Clin Oncol $2018 ; 36: 2872-8$.

50. Haratani K, Hayashi $\mathrm{H}$, Chiba $\mathrm{Y}$, et al. Association of immune-related adverse events with nivolumab efficacy in non-small-cell lung cancer. JAMA Oncol $2018 ; 4: 374-8$

\section{TIRÉS À PART}

M. Kostine

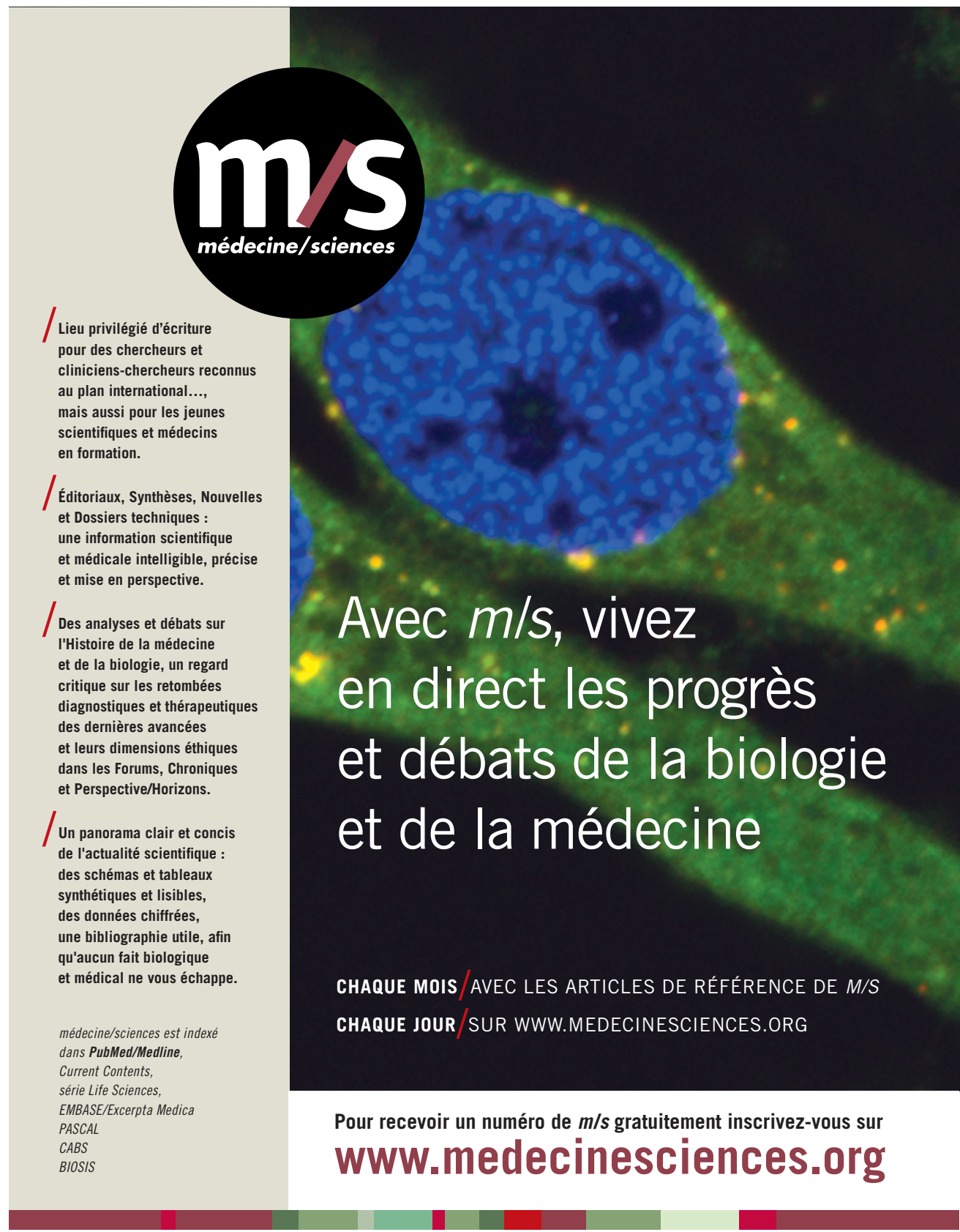

